\title{
Reduction in the processing of possible blood culture contaminants by the application of a selection criterion
}

\author{
IOANNIS K. NEONAKIS ${ }^{1}$, DIMITRA STAFYLAKI ${ }^{1}$ and DEMETRIOS A. SPANDIDOS ${ }^{2}$
}

${ }^{1}$ Department of Clinical Microbiology and Microbial Pathogenesis, University Hospital of Heraklion, 71201 Heraklion; ${ }^{2}$ Laboratory of Clinical Virology, School of Medicine, University of Crete, 71003 Heraklion, Greece

Received May 20, 2020; Accepted June 23, 2020

DOI: $10.3892 / \mathrm{etm} .2020 .8935$

\begin{abstract}
Possible blood culture (BC) contaminants are generally considered to be skin flora species including coagulase-negative Staphylococci (CNS), Corynebacterium species, Micrococcus species, Bacillus species and Propionibacterium acnes. Prior to October 1, 2016 all possible BC contaminants were fully processed (identification, susceptibility testing) in our laboratory. In order to reduce the laboratory workload from October 1, 2016 a possible contaminant was only processed if it was present in more than one BC pair drawn from the same patient within the same day. The two-year study period was divided in two periods namely period A from January 1, 2016 to September 30, 2016 (first 9 months) and period B from October 1, 2016 to December 31, 2017 (last 15 months). A series of indices (INs) were calculated including among others the Working Rate IN (WR) defined as the total isolates divided to the total number of BCs submitted per month and the CNS Rate (CNSR) defined as the total number of CNS processed divided to the total number of BCs submitted per month. A $23.08 \%$ reduction in the CNSR was noted (from $3.51 \%$ in period $\mathrm{A}$ to $2.70 \%$ in period $\mathrm{B}$ ) whereas the overall WR was reduced from $7.19 \%$ in period A to $6.84 \%$ in period B. Furthermore, the total number of contaminants processed per month divided to the total number of isolates processed per month was reduced from $54.50 \%$ in period A to $42.41 \%$ in period $\mathrm{B}$. The reduction in the INs recorded is of great value since it was achieved by the implementation of a simple criterion easily applicable and without any cost.
\end{abstract}

Correspondence to: Dr Ioannis K. Neonakis, Department of Clinical Microbiology and Microbial Pathogenesis, University Hospital of Heraklion, 71201 Heraklion, Greece

E-mail: ineonakis@gmail.com

Key words: blood cultures, contaminants, coagulase-negative Staphylococci, Corynebacterium species, Micrococcus species, Bacillus species, Propionibacterium acnes

\section{Introduction}

Blood cultures (BCs) are the gold standard method for the diagnosis of bacteremias. A BC isolate is considered to be a contaminant when it is not pathogenic for a patient and is introduced into the culture of the patient during either blood collection or processing $(1,2)$. Among others, contaminated BCs lead to unnecessary administration of antibiotics, prolonged hospital stay, unnecessary removal of central intravenous lines, increased work load and cost.

In the present study we aimed to evaluate the effect in the laboratory work load by stopping the processing (identification, susceptibility testing) of possible contaminant isolates by the application of a certain exclusion criterion.

\section{Materials and methods}

All BCs sent to our Laboratory from January 1, 2016 to December 31, 2017 were analyzed. All positive BCs were processed according to standard protocols and identification and susceptibility testing of the isolates were performed with Vitek2 (BioMerieux, France). Only the first isolate per species and patient was included in the study. Since the majority of the samples from the Orthopedic Department were pusses or non sterile body fluids inoculated in $\mathrm{BC}$ vials, all isolates from this particular Department were excluded from the analysis. As possible contaminants (from now on referred to as contaminants) were considered skin flora species including coagulase-negative Staphylococci (CNS), Corynebacterium species (sp.), Micrococcus sp., Bacillus sp. and Propionibacterium acnes. All viridians group Streptococci were always fully processed.

The two-year time period was divided into two parts: period A from January 1, 2016 to September 30, 2016 (first 9 months) and part B from October 1, 2016 to December 31, 2017 (last 15 months). During the first period all isolates including all contaminants were processed (identification/susceptibility testing) whereas in the second period among contaminants only those meeting a selection criterion were processed. This selection criterion was the presence of the same contaminant in more than one BC pairs drawn from the patient within the same day. After discussion and agreement with the clinicians a series of Departments were excluded from the application of the criterion including all Pediatric Wards, all Wards handling cancer patients along with the Nephrology and Neurology Departments. 
Table I. Results of period A (January 2016 - September 2016).

\begin{tabular}{lrrrrrrrrrr}
\hline \multirow{2}{*}{ Variables } & $\begin{array}{c}\text { January } \\
2016\end{array}$ & $\begin{array}{c}\text { February } \\
2016\end{array}$ & $\begin{array}{c}\text { March } \\
2016\end{array}$ & $\begin{array}{c}\text { April } \\
2016\end{array}$ & $\begin{array}{c}\text { May } \\
2016\end{array}$ & $\begin{array}{c}\text { June } \\
2016\end{array}$ & $\begin{array}{c}\text { July } \\
2016\end{array}$ & $\begin{array}{c}\text { August } \\
2016\end{array}$ & $\begin{array}{c}\text { September } \\
2016\end{array}$ & $\begin{array}{r}\text { Total } \\
\text { Per A }\end{array}$ \\
\hline CNS & 45 & 51 & 30 & 30 & 44 & 50 & 38 & 48 & 49 & 385 \\
Bacillus sp. & 0 & 0 & 2 & 2 & 1 & 2 & 0 & 1 & 1 & 9 \\
Coryne sp. & 0 & 0 & 1 & 2 & 1 & 3 & 2 & 3 & 3 & 15 \\
Micrococcus sp. & 1 & 1 & 2 & 3 & 0 & 1 & 1 & 0 & 0 & 9 \\
Propionibacterium acnes & 0 & 1 & 0 & 1 & 0 & 4 & 3 & 1 & 2 & 12 \\
Total contaminants & 46 & 53 & 35 & 38 & 46 & 60 & 44 & 53 & 55 & 430 \\
Total bottles & 1,378 & 1,292 & 1,075 & 1,230 & 1,183 & 1,182 & 1,124 & 1,267 & 1,249 & 10,980 \\
Total isolates & 80 & 94 & 62 & 85 & 82 & 101 & 81 & 109 & 95 & 789 \\
Working rate (\%) & 5.81 & 7.28 & 5.77 & 6.91 & 6.93 & 8.54 & 7.21 & 8.60 & 7.61 & 7.19 \\
Contaminant rate (\%) & 3.34 & 4.10 & 3.26 & 3.09 & 3.89 & 5.08 & 3.91 & 4.18 & 4.40 & 3.92 \\
CNS rate (\%) & 3.27 & 3.95 & 2.79 & 2.44 & 3.72 & 4.23 & 3.38 & 3.79 & 3.92 & 3.51 \\
C/I ratio (\%) & 57.50 & 56.38 & 56.45 & 44.71 & 56.10 & 59.41 & 54.32 & 48.62 & 57.89 & 54.50 \\
\hline
\end{tabular}

C/I, contaminants/isolates; CNS, coagulase-negative Staphylococci; Per, period; sp., species.

Moreover, in all cases of positive BCs with more than one isolate, including contaminant and non-contaminant isolates, all contaminants were fully processed and included in the analysis.

A series of indices (INs) were calculated. In particular: i) the Working Rate IN (WR) defined as the total isolates divided to the total number of BCs submitted per month; ii) the Contaminant Rate IN (CR) defined as the total number of contaminants processed divided to the total number of BCs submitted per month; iii) the CNS Rate (CNSR) defined as the total number of CNS processed divided to the total number of BCs submitted per month; and iv) the C/I Ratio defined as the total number of contaminants processed per month divided to the total number of isolates processed per month.

\section{Results}

Over the whole period of the two years a total of 31,579 bottles were admitted and the total isolates were 2,199 (total WR: $6.96 \%$ ). The total contaminants of the period were 1,028 (total CR: $3.26 \%$ ) including $941 \mathrm{CNS}$ (total CNSR: 2.98\%), 23 Bacillus sp., 33 Corynebacterium sp., 19 Micrococcus sp., and 12 Propionibacterium acnes. CNS comprised $42.79 \%$ of the total 2,199 isolates, whereas Bacillus sp. comprised $1.05 \%$, Corynebacterium sp. $1.5 \%$, Micrococcus sp. $0.86 \%$, and Propionibacterium acnes $0.55 \%$ of the total isolates. The overall $\mathrm{C} / \mathrm{I}$ ratio of the period was $46.75 \%$.

The species distribution, the number of bottles admitted, the number of isolates and contaminants processed along with the WR, the CR, the CNSR and the C/I Ratio per month and as total for period A and period B are shown in Tables I and II, respectively.

\section{Discussion}

$\mathrm{BCs}$ remain the major tool for the diagnosis of bacteremias. Bacteremias are a significant cause of morbidity and mortality.
Mortality can reach $37 \%$ as previously reported (3). BC contaminations are not uncommon. Despite the standard recommendation for $3 \% \mathrm{BC}$ contamination rate usually the reports are above this level and vary significantly even reaching $12 \%(4,5)$. The high fluctuation in $\mathrm{BC}$ contamination rate reported is partially due to the lack of uniformity since some authors decide themselves whereas others rely on the contributing microbiologist's decision on the contaminant status of an isolate (4). Despite this, the contamination rate is a significant indication of the quality provided by the hospital health-care. In the present study the overall contamination rate over the two-year period was found to be $3.26 \%$, which is higher from the analogous indice of $2.7 \%$ previously reported from Greece (6). The overall CNS rate was $2.98 \%$. CNS comprised $42.79 \%$ of total isolates, which is a slightly higher percentage than that found from a previous study in Greece $(35.9 \%)(7)$.

Apart from the major clinical impact of BC contaminations and the relevant alteration in the clinical treatment algorithms, the overall additional cost burden due to $\mathrm{BC}$ contaminations is very high and is estimated as a median of $\$ 8,720$ (8). Among others this cost is due to increased length of stay (estimated as a median 4.5-day increase in North America), increased microbiology laboratory charges and increased antibiotic use $(4,5)$. In the first nine months of the present study all isolates including all possible contaminants were fully processed thus allowing the clinicians to decide themselves whether the presence of a possible contaminant in the blood was of clinical importance or not. Over the last fifteen months of the present study and in order to reduce the microbiology laboratory cost possible contaminants were only processed if they met the criterion of their presence in a second BC set of the same patient within the same day. The implementation of this criterion was easy, costless and most of all safe for the patients since the clinicians were informed immediately and their consensus was required. 


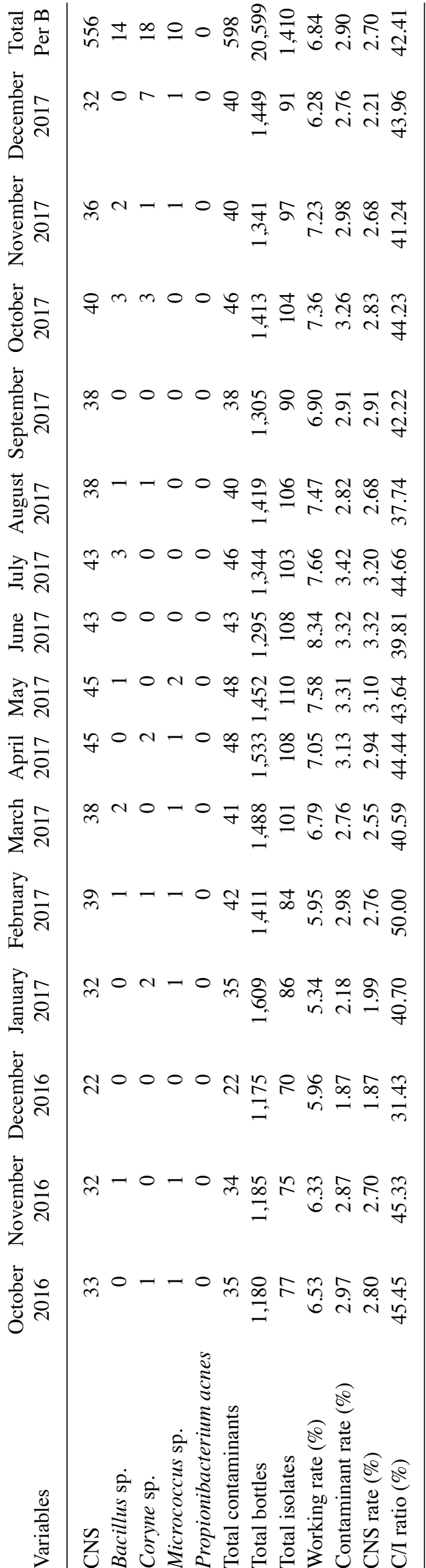

In the present study, when comparing the two periods a $26.02 \%$ reduction in the CR was found (from $3.92 \%$ in period A to $2.90 \%$ in period B). An analogous percentage was recorded in the reduction of the CNSR (23.08\%; from $3.51 \%$ in period A to $2.70 \%$ in period $\mathrm{B}$ ). The overall WR was reduced from $7.19 \%$ in period $A$ to $6.84 \%$ in period $B$ and furthermore, the $\mathrm{C} / \mathrm{I}$ Ratio was reduced from $54.50 \%$ in period A to $42.41 \%$ in period $\mathrm{B}$. The reduction in all INs noted is of great value since it was achieved by the implementation of a simple, easily applicable criterion and without any cost. If more wards of the Hospital were to be included in the application of the criterion an even higher reduction would be expected and such an application is strongly advocated.

\section{Acknowledgements}

Not applicable.

\section{Funding}

No funding was received.

\section{Availability of data and materials}

Not applicable.

\section{Authors' contributions}

IKN and DS carried out the experiments. DAS, IKN and DS wrote the manuscript with input from each author, and approved the final manuscript.

\section{Ethics approval and consent to participate}

Not applicable.

\section{Patient consent for publication}

Not applicable.

\section{Competing interests}

DAS is the Editor-in-Chief for the journal, but had no personal involvement in the reviewing process, or any influence in terms of adjudicating on the final decision, for this article. The other authors declare that they have no competing interests.

\section{References}

1. Hall KK and Lyman JA: Updated review of blood culture contamination. Clin Microbiol Rev 19: 788-802, 2006.

2. Altindis M, Koroglu M, Demiray T, Dal T, Ozdemir M, Sengil AZ, Atasoy AR, Doğan M, Cicek AC, Ece G, et al: A multicenter evaluation of blood culture practices, contamination rates, and the distribution of causative bacteria. Jundishapur $\mathbf{J}$ Microbiol 9: e29766, 2016.

3. Long B and Koyfman A: Best clinical practice: Blood culture utility in the emergency department. J Emerg Med 51: 529-539, 2016.

4. Hughes JA, Cabilan CJ, Williams J, Ray M and Coyer F: The effectiveness of interventions to reduce peripheral blood culture contamination in acute care: A systematic review protocol. Syst Rev 7: 216, 2018 
5. El Feghaly RE, Chatterjee J, Dowdy K, Stempak LM, Morgan S, Needham W, Prystupa K and Kennedy M: A quality improvement initiative: Reducing blood culture contamination in a Children's Hospital. Pediatrics 142: e20180244, 2018.

6. Hadziyannis AS, Stephanou I, Dimarogona K, Pantazatou A, Fourkas D, Filiagouridis D and Avlami A: Blood culture results during the period 1995-2002 in a Greek tertiary care hospital. Clin Microbiol Infect 10: 667-670, 2004.

7. Falagas ME, Bakossi A, Pappas VD, Holevas PV, Bouras A and Stamata E: Secular trends of blood isolates in patients from a rural area population hospitalized in a tertiary center in a small city in Greece. BMC Microbiol 6: 41, 2006.
8. Gander RM, Byrd L, DeCrescenzo M, Hirany S, Bowen M and Baughman J: Impact of blood cultures drawn by phlebotomy on contamination rates and health care costs in a hospital emergency department. J Clin Microbiol 47: 1021-1024, 2009.

(i) $(-)$ This work is licensed under a Creative Commons Attribution-NonCommercial-NoDerivatives 4.0 International (CC BY-NC-ND 4.0) License. 